\title{
Utility of 18-Fluorodeoxyglucose positron emission tomography in the
}

\section{prediction of ventricular tachycardia and advanced conduction disease in}

\section{patients with cardiac sarcoidosis}

Roy Chung1, Wilson Tang1, Richard C. Brunken², Gian Novaro ${ }^{2}$, Daniel Culver $^{3}$, and Patrick J. Tchou ${ }^{1}$

1. The Department of Cardiovascular Medicine, Heart and Vascular Institute, Cleveland Clinic, Cleveland, Ohio, USA

2. The Department of Molecular and Functional Imaging, Cleveland Clinic, Cleveland, Ohio, USA

3. The Department of Pulmonary, Allergy and Critical Care Medicine, Cleveland Clinic, Cleveland, Ohio, USA

\section{RESEARCH}

Please cite this paper as: Chung R, Tang WHW, Brunken RC, Culver D, Tchou PJ. Utility of 18-Fluorodeoxyglucose positron emission tomography in the prediction of ventricular tachycardia and advanced conduction disease in patients with cardiac sarcoidosis. AMJ 2018;11(8):410-420 https://doi.org/10.21767/AMJ.2018.3218

\section{Corresponding Author:}

Roy Chung, MD

Department of Cardiovascular Medicine

Heart and Vascular Institute, Cleveland Clinic

Cleveland, Ohio, USA

Email: chungr@ccf.org

\section{ABSTRACT}

\section{Background}

The diagnosis and management of cardiac sarcoidosis (CS) along with the recognition of high risk features remains a significant challenge. Failing to identify reliable disease prognosticators often results in fatal ventricular arrhythmia or heart failure.

\section{Aims}

This study sought to determine the association of regional fluorodeoxyglucose positron emission tomography $\left({ }^{18} \mathrm{FDG}\right.$ PET) patterns and the presence of ventricular tachycardia (VT) storm and conduction disease in patients with CS.

\section{Methods}

In an observational cohort analysis, patients with a working diagnosis of CS who underwent ${ }^{18}$ FDG PET imaging were included. Baseline clinical data and ${ }^{18}$ FDG PET data were collected and analysed.

\section{Results}

A total of 57 patients with CS were studied. All had abnormal ${ }^{18}$ FDG PET imaging consisting of inflammation, scar or combination of both. VT storm was present in 33 per cent of the cohort, while 63 per cent had high grade conduction disease. Mean inflammatory burden noted on ${ }^{18}$ FDG PET in those with VT storm was 12 per cent. Inflammation involving $\geq 2$ basal septal or mid septal segments ( $>15$ per cent myocardium) was an independent predictor of VT storm (OR: 9.62, 95 per cent Cl 1.97-46.9, $p=0.005)$. The degree of inflammatory burden and regional inflammation pattern on ${ }^{18}$ FDG PET demonstrated significant concordance with the presentation of VT storm. Inflammation in the anteroseptal segment was associated with high grade conduction disease (OR: 8.00, 95 per cent $\mathrm{Cl}$ 0.94-68, $p=0.029$ )

\section{Conclusion}

The presence of $>15$ per cent myocardial inflammation on ${ }^{18}$ FDG PET imaging and regional inflammation $\geq 2$ basal or mid septal segment significantly correlated with risk of ventricular arrhythmia specifically VT storm regardless of scar burden.

\section{Key Words}

FDG PET imaging, cardiac sarcoidosis, ventricular arrhythmias, conduction disease

\section{What this study adds:}

\section{What is known about this subject?}

The diagnosis of cardiac sarcoidosis amongst individuals with ventricular tachycardia or atrioventricular heart block remains challenging. 


\section{What new information is offered in this study?}

Significant regional inflammation on ${ }^{18}$ FDG PET imaging is associated with ventricular tachycardia storm.

3. What are the implications for research, policy, or practice?

We recommend ${ }^{18}$ FDG PET be incorporated as an adjunct imaging modality for risk stratification and management in patients with cardiac sarcoidosis.

\section{Background}

Sarcoidosis is an inflammatory disorder characterized by granulomatous deposits occurring in the pulmonary parenchyma, lymph nodes, myocardium, liver, spleen, nervous system and other tissues. Cardiac involvement has been reported as high as 25 per cent among patients with sarcoidosis in the United States. ${ }^{1-4}$ A Finnish 25 year study yielded an annual detection rate of 0.31 per $10^{5}$ adults with a prevalence of 2.2 per $10^{5}$, which remains a clinical rarity. ${ }^{5}$ Although patients with cardiac sarcoidosis (CS) may have an early indolent course, fatal ventricular arrhythmias, symptomatic bradycardia or fulminant heart failure may ensue leading to poor survival. Kandolin et al. demonstrated in their series of CS patients, $44 \%$ presented with significant conduction disease, $33 \%$ with ventricular arrhythmias and $18 \%$ with heart failure. ${ }^{5}$

The diagnosis of CS remains challenging among pulmonary and cardiovascular clinicians. Heart Rhythm Society has incorporated the role of 18-fluorodeoxyglucose positron emission tomography $\left({ }^{18}\right.$ FDG PET) in the diagnosis of CS but not its management. ${ }^{6}$ The use of ${ }^{18}$ FDG PET has aided in identifying cardiac involvement of sarcoidosis and in the assessment of disease activity. ${ }^{7} \quad$ Nonetheless, the association between ${ }^{18}$ FDG PET imaging findings and the occurrence of arrhythmic events including conduction disease and malignant ventricular arrhythmia in patients with established CS has not been well described. Thus, the objective of our study was to identify and test the utility of ${ }^{18}$ FDG PET imaging patterns and their association with high grade atrioventricular (AV) conduction disease and with ventricular tachycardia (VT) in patients with CS.

\section{Method}

\section{Patient population}

Between January $1^{\text {st }} 2009$ and December $31^{\text {st }} 2011$, consecutive patients with a diagnosis of $\mathrm{CS}$, who underwent ${ }^{18}$ FDG PET imaging at our institution and evaluated at the cardiology and pulmonary clinics at Cleveland Clinic, Cleveland, $\mathrm{OH}$ were included in the study. Patients with significant coronary artery disease were excluded from the study. Among the 57 patients with CS, 10 were cardiac biopsy proven, 39 were extra-cardiac biopsy proven and met 2 of the 4 major criteria as specified by the 2006 revised Japanese Ministry of Health and Welfare guidelines for the diagnosing CS, while the remaining 8 had abnormal cardiac ${ }^{18}$ FDG PET imaging, specifically inflammatory changes associated with ventricular arrhythmias and hilar adenopathy (Figure 1). Demographic, clinical, echocardiographic, ${ }^{18}$ FDG PET imaging and electrocardiographic data, implantable cardioverter defibrillator (ICD) or pacemaker implantation, antiarrhythmic drug (AAD) use, and the presence of immunosuppressive therapy were collected. Hospitalization records were reviewed for outcomes. The study was approved by the Cleveland Clinic institutional review board and ethics committee and performed in accordance with institutional guidelines.

\section{${ }^{18}$ FDG PET evaluation protocol}

All patients underwent baseline electrocardiographic and echocardiographic examinations. Patients with suspected CS based upon clinical presentation including conduction disease and ventricular arrhythmia or echocardiographic abnormality prospectively underwent further testing, including cardiac magnetic resonance imaging, and/or ${ }^{18}$ FDG PET imaging. We ask our patients to consume a low carbohydrate meal the night before imaging. The patients are asked to fast for a minimum of 12 hours prior to the FDG injection. We do not use low dose heparin to raise free fatty acid levels. We routinely check a blood glucose level at the time of imaging, but do not use these levels to determine if we should proceed with imaging, as some with diabetes or insulin resistance due to steroids may have higher levels than those without these disorders. We are able to assess the adequacy of switching to fatty acid utilization in striated muscle by looking at the uptake of FDG in the psoas muscle on the whole body images. If FDG uptake in the psoas muscle is low, we believe that the appropriate switch in metabolism from glucose to free fatty acid has occurred. The imaging protocol included gated resting perfusion and fasting ${ }^{18}$ FDG PET whole body scan. Images were obtained using a 64-slice PET-computed tomography scanner (Siemens Biograph Truepoint PET-CT). Patients underwent cardiac resting perfusion scan using either Rubidium-82 or Nitrogen-13 ammonia. ${ }^{18}$ FDG was subsequently infused to acquire whole body and cardiac images. Low dose computed tomography scans without contrast were performed for attenuation correction. Cardiac PET images were evaluated and reconstructed to standard short-axis, horizontal long-axis and vertical short- 
axis views. Registration between the cardiac PET and computed tomography images were performed using cardiac and extra-cardiac anatomical landmarks. Cardiac biopsies were attempted if at all possible if the diagnosis was equivocal. Cardiac angiography was performed in all patients to rule out significant obstructive coronary artery disease.

${ }^{18}$ FDG PET imaging patterns were described using the standard American Heart Association 17 segment model, and interpreted into 4 different categories depending on perfusion and FDG uptake abnormalities. In normal studies, there is normal perfusion and no FDG uptake. In the absence of perfusion but increased FDG uptake in the corresponding myocardial segment, the study would be consistent with inflammation. In the absence of perfusion and FDG uptake, the study would suggest myocardial scar. In some studies, there was a combination of inflammation and scar in various myocardial segments. For the purpose of this study, the terms inflammation and scar will be used to correspond to the respective ${ }^{18}$ FDG PET imaging results.

\section{Arrhythmia definitions}

In our study cohort, VT storm was clinically defined as patients with more than two episodes of sustained VT necessitating intravenous AADs such as amiodarone or lidocaine, or patients who received more than three episodes of ICD anti-tachycardia pacing or shocks within a 24 hour period. Those who had evidence of VT but did not meet these criteria were categorized as having nonsustained VT. In reference to high grade conduction disease, we categorized significant conduction disease as Mobitz 2 AV block, bifascicular block, complete AV block or trifasficular block.

\section{Statistical analysis}

Baseline characteristics were described with means and standard deviations. Comparisons between study groups, as they relate to patients with and without VT storm and patients with and without conduction disease were made using t-test and chi square, for continuous and categorical variables, as appropriate. Logistic regression was utilized in order to control for confounders. Odds ratios (OR) were reported with their respective 95 per cent confidence intervals $(\mathrm{Cl})$. Receiver operating characteristic curves were used to establish an optimum threshold for the association between ${ }^{18}$ FDG PET findings and prediction of arrhythmic events. Multivariate analysis was developed to determine the association of various ${ }^{18}$ FDG PET imaging patterns and other clinical variables and the chance of developing VT storm and conduction disease. When a certain ${ }^{18}$ FDG PET imaging pattern was found to have high correlation coefficient to VT storm, linear regression line was then constructed based on this ${ }^{18}$ FDG PET imaging finding in order to estimate the risk of VT storm in this population. Probability of VT storm risk was plotted as a function of ${ }^{18}$ FDG PET inflammation. By multiple analyses of variance, a linear regression model was found to be optimum and statistically significant. As a result, an equation was derived for the prediction of VT risk depending on the ${ }^{18}$ FDG PET inflammatory burden (VT risk=0.1579+0.01487 x percentage of inflammation). $P$ values of less than 0.05 were considered statistically significant. The statistical analysis was performed using SPSS (version 16) and MedCalc (version 12).

\section{Results}

Baseline clinical characteristics of the study population $(n=57)$ are shown in Table 1 . CS patients were predominantly Caucasian (81 per cent), middle-aged (49 \pm 12 years), and with an equal gender distribution; mean left ventricular ejection fraction was $0.38 \pm 0.15$. None of the patients had significant obstructive coronary artery disease by coronary angiography. Seventy percent of patients had non-sustained VT, 33 per cent had VT storm while 63 per cent had significant conduction disease. Almost all patients (98 per cent) had abnormal ${ }^{18}$ FDG PET imaging scans, regardless of pattern of inflammation, scar or combination of both.

\section{VT storm}

Patients with VT storm tended to have a positive cardiac biopsy (37 per cent vs. 8 per cent, $p=0.03$ ), and be on either Class 1B (26 per cent vs. 3 per cent, $p=0.006$ ) or Class 3 AADs (63 per cent vs. 21 per cent, $\mathrm{p}=0.002$ ) (Table 2). VT storm patients tended to be on left ventricular assist devices or be cardiac transplant recipients (32 per cent vs. 2.6 per cent, $p=0.002$ ). The use of immunosuppressive agents such as corticosteroids, methotrexate, leflunomide and infliximab did not differ between the 2 groups, although mycophenolate was used more often in the former group. The mean inflammatory burden noted by ${ }^{18}$ FDG PET in this cohort was 12 per cent of the myocardium. Among the 4 patients who lacked tissue biopsy for the diagnosis of CS, the mean inflammatory burden noted on ${ }^{18}$ FDG PET was 23 per cent of the myocardium. In general, the finding of inflammation or scar, or the combination of both on ${ }^{18} \mathrm{FDG}$ PET imaging was similar in both groups. Regional inflammation in $\geq 2$ basal or mid septal segments was a significant independent predictor of VT storm, after adjusting for age, gender, race, and left ventricular ejection 
fraction (OR: 9.62, 95 per cent $\mathrm{Cl}$ 1.97-46.9, $\mathrm{p}=0.005$, Figure 2).

\section{Inflammation burden on ${ }^{18}$ FDG PET scan}

Quantitative measure of the number of segments with inflammation on ${ }^{18}$ FDG PET imaging correlated with a higher occurrence of VT storm. The risk of VT storm was noted to be 48 per cent when 2 segments were involved, compared to 67 per cent when 5 segments were involved. By receiver operating characteristic curve analysis in an estimated sample size of 60 patients, relative inflammation on ${ }^{18} \mathrm{FDG}$ PET greater than 15 per cent involvement of the left ventricle predicted VT storm with an area under the curve of 0.68 (95 per cent $\mathrm{Cl} 0.55-0.80, \mathrm{p}=0.034$ ), and resulted in a sensitivity of 63 per cent, specificity of 84 per cent, positive predictive value of 67 per cent, and negative predictive value of 82 per cent (Figure 3). Patients with a greater than 15 per cent mismatch had more than six times the risk of VT storm (OR: 6.73, 95 per cent $\mathrm{Cl}$ 1.92-23.6, $p<0.01)$. The observed risk of VT storm, as a function of inflammatory burden, was compared to our predicted risk of VT storm utilizing our predictor equation. We found good correlation between the observed and predicted risk of VT storm, albeit our equation tended to underestimate risk by 8 per cent-18 per cent (Figure 4). It should be noted, however, that the equation developed is limited by the small sample size and the exponential relationship between inflammation and VT risk after 6 per cent involvement (Table 3).

\section{Conduction disease}

Patients with high grade AV conduction disease tend to be older, with a mean left ventricular ejection fraction of $0.37 \pm 0.16$. Thirty nine per cent $(n=14 / 36)$ had complete heart block, 11 per cent $(n=4 / 36)$ had 2:1 AV block, and 50 per cent $(n=18 / 36)$ had bifascicular block. Although the general finding of inflammation, scar or the combination of both did not discriminate patients within this cohort, in a multivariate regression model adjusted for confounders such as age, gender, race, and ejection fraction, there was a significant association among those with ${ }^{18}$ FDG PET inflammation in the anteroseptal region and high grade AV conduction disease (OR: 8.00, 95 per cent $\mathrm{Cl}$ 0.94-68, $p=0.029$, Figure 2).

\section{Immunosuppression therapy}

Our immunosuppression protocols were based on the patient's clinical presentation. In many cases of VT storm, all patients received a 3-5 day course of 1gram methylprednisolone followed by prednisone $40 \mathrm{mg}$ daily and subcutaneous methotrexate. All VT storm abated following pulse steroid infusions. In cases of recurrences of sustained VT or frequent ventricular ectopies while on high dose prednisone, these individuals progressed to either weekly infliximab infusion or steroid sparing agents such as leflunomide. ${ }^{18}$ FDG PET imaging was repeated at 3-6 monthly intervals to evaluate disease activity as relapses of ventricular arrhythmias were common during immunosuppression tapering. Of the 57 patients, 20 had follow up ${ }^{18}$ FDG PET imaging during their clinical course with a mean follow up period of $401 \pm 518$ days. A mean diminution of $8.9 \pm 8.2$ per cent inflammation of myocardium was noted on subsequent ${ }^{18}$ FDG PET scans following immunosuppression. Patients with VT storm $(n=7)$ had a greater reduction of inflammation burden (11 per cent vs. 8 per cent of myocardium) compared to those without VT storm. For all available follow up ${ }^{18}$ FDG PET studies, resolution of inflammatory regions seen on initial scan were replaced with scar on follow up studies.

\section{Discussion}

In this study of patients with suspected CS who were evaluated with ${ }^{18}$ FDG PET imaging, regional myocardial inflammatory abnormalities were associated with VT storm and high grade conduction disease. The principal finding was the predictive value of $>15$ per cent myocardial inflammation on ${ }^{18}$ FDG PET imaging and regional inflammation $\geq 2$ basal or mid septal segments correlating with the risk of malignant ventricular arrhythmia regardless of scar burden.

The pathogenesis of CS involves two critical distinct phases. In the initial phase, sarcoid infiltration consisting of mononuclear phagocytes and CD4 positive $T$ cells elicit interleukin-2 and interferon-gamma which results in local inflammation, hence giving rise to perfusion metabolism mismatch with an increased FDG uptake in areas with lack of perfusion on ${ }^{18}$ FDG PET. As the disease evolves into a later phase, $\mathrm{T}$ helper type 2 response pathways produce anti-inflammatory cytokines such as interleukin-10 and transforming growth factor promoting fibrous tissue formation, resulting in fibrosis noted as a matched metabolism perfusion defect on ${ }^{18}$ FDG PET. ${ }^{8}$

Cardiac magnetic resonance imaging has been proposed as a potential and complementary imaging tool in $\mathrm{CS}^{9-11}$ The sensitivity in detecting CS is comparable to ${ }^{18}$ FDG PET imaging when this was evaluated by Smedema et al., although the specificity was lower in ${ }^{18}$ FDG PET imaging. ${ }^{12}$ Greulich et al. recently demonstrated that myocardial scar detected by late gadolinium enhancement was the best independent predictor of mortality, appropriate ICD 
discharges and aborted sudden cardiac death, confirming previous work done by Patel et al. ${ }^{13,14}$ However the presence of pacemaker or ICD which are common in this population poses a challenge due to the potential of the magnetic field causing localized heating of leads and device reprogramming. This may be overcome by newly emerging magnetic resonance-compatible pacing systems although similarly compatible ICDs are not yet available..$^{9-11,14,15}$ This limitation alone prohibits the routine use of magnetic resonance imaging as an imaging tool in the surveillance and management of CS disease activity with currently implanted cardiac devices. Finally, the current American Heart Association scientific statement discourages the use of cardiac magnetic resonance imaging in patients with permanent cardiac pacemakers or defibrillators unless there is a strong clinical indication where its benefits outweigh the risks or an alternative test is unavailable. ${ }^{16}$

Early identification of CS in patients with ventricular arrhythmias is proven to be crucial as they invariably need high doses of immunosuppression to either halt a worsening of their myocardial function or terminate their arrhythmias. ${ }^{18}$ FDG PET imaging has been proposed to be the imaging test of choice in CS, reported in a recent metaanalysis by Yousef et al. ${ }^{7,17,18}$ Blankstein et al. concluded inflammation in ${ }^{18}$ FDG PET imaging identified patients with suspected cardiac sarcoidosis is at higher risk for VT or death. In our series however, we further quantified and demonstrated significant association between regional inflammatory changes of $\geq 2$ basal or mid septal segments or the presence of $\geq 2$ segments of inflammation on ${ }^{18}$ FDG PET imaging and VT storms. Patients with CS with such imaging findings, may be prompted to initiation of immunosuppression prior to unnecessary ICD therapies or VT. Furthermore, intra-cardiac and extra-cardiac inflammation noted on ${ }^{18}$ FDG PET may be instrumental as reference points in follow up imaging to determine response to systemic immunosuppression. ${ }^{19}$

The association of impaired left ventricular ejection fraction or scar with ventricular arrhythmias has been widely acknowledged. ${ }^{19-21}$ Thus, we tried to evaluate if scar burden on ${ }^{18}$ FDG PET or ejection fraction may have an association with non-sustained VT or VT storm. However among patients with ejection fraction $<40$ per cent or scar noted on ${ }^{18}$ FDG PET, there was no significant association between non-sustained VT and VT storm. This further emphasizes the utility of specific regional inflammatory changes on ${ }^{18} \mathrm{FDG}$ PET imaging and their association with ventricular arrhythmias.

\section{Study limitations}

The current study is a single-centre, nonrandomized, observational study in a tertiary care centre which inherently incorporates a referral bias. As such, there may be residual confounding factors that remain despite careful adjustment of significant covariates. The small sample size, coupled with several patients lacking histologic confirmation can only result in a hypothesis generating analysis in regards to the earlier detection of CS and the importance of aggressive immunosurpression. Despite the high cost of ${ }^{18}$ FDG PET studies and its associated radiation, its use in this subgroup of critically ill patients has certain proven value and can invariably impact their long term outcome.

\section{Conclusion}

CS continues to pose diagnostic challenges especially in the occult stage. Importantly; the degree of inflammatory burden and regional inflammation on ${ }^{18}$ FDG PET demonstrates significant concordance with VT storm. The threshold for these arrhythmias is lower when $>2$ myocardial segments of inflammation are noted on ${ }^{18} \mathrm{FDG}$ PET, or if inflammation involves $\geq 2$ basal or mid septal segments. Patients with high grade conduction disease were noted to have anteroseptal segmental inflammation. We recommend that ${ }^{18}$ FDG PET be incorporated as an adjunct imaging modality for risk stratification and management of CS patients.

\section{References}

1. Hillerdal G, Nou E, Osterman K, et al. Sarcoidosis: Epidemiology and prognosis. A 15-year European study. Am Rev Respir Dis. 1984;130:29-32.

2. Rybicki BA, Maliarik MJ, Major M, et al. Epidemiology, demographics, and genetics of sarcoidosis. Semin Respir Infect. 1998;13:166-73.

3. Reich JM. Re: "racial differences in sarcoidosis incidence: A 5-year study in a health maintenance organization". Am J Epidemiol. 1998;148:100-01.

4. Westney GE, Judson MA. Racial and ethnic disparities in sarcoidosis: From genetics to socioeconomics. Clin Chest Med. 2006;27:453-62.

5. Kandolin R, Lehtonen J, Airaksinen J, et al. Cardiac sarcoidosis: epidemiology, characteristics, and outcome over 25 years in a nationwide study. Circulation. 2015;131(7):624-32.

6. Birnie DH, Sauer WH, Bogun F, et al. HRS expert consensus statement on the diagnosis and management of arrhythmias associated with cardiac sarcoidosis. Heart Rhythm. 2014;11(7):1305-23.

7. Yamagishi $H$, Shirai N, Takagi $M$, et al. Identification of 
cardiac sarcoidosis with (13)N-NH(3)/(18)F-FDG PET. J Nucl Med. 2003;44:1030-6.

8. Doughan AR, Williams BR. Cardiac sarcoidosis. Heart. 2006;92:282-8.

9. Riedy K, Fisher MR, Belic $N$, et al. MR imaging of myocardial sarcoidosis. AJR Am J Roentgenol. 1988;151:915-6.

10. Matsuki $M$, Matsuo M. MR findings of myocardial sarcoidosis. Clin Radiol. 2000;55:323-5.

11. Ordovas KG, Higgins CB. Delayed contrast enhancement on MR images of myocardium: Past, present, future. Radiology. 2011;261:358-74.

12. Smedema JP, White L, Klopper AJ. FDG-PET and MIBI-tc SPECT as follow-up tools in a patient with cardiac sarcoidosis requiring a pacemaker. Cardiovasc J Afr. 2008;19:309-10.

13. Greulich S, Deluigi CC, Gloekler S, et al. CMR imaging predicts death and other adverse events in suspected cardiac sarcoidosis. JACC Cardiovasc Imaging. 2013;6:501-11.

14. Patel MR, Cawley PJ, Heitner JF, et al. Detection of myocardial damage in patients with sarcoidosis. Circulation. 2009;120:1969-77.

15. Wilkoff BL, Bello D, Taborsky $M$, et al. Magnetic resonance imaging in patients with a pacemaker system designed for the magnetic resonance environment. Heart Rhythm. 2011;8:65-73.

16. Levine GN, Gomes AS, Arai AE, et al. Safety of magnetic resonance imaging in patients with cardiovascular devices: An American Heart Association scientific statement from the committee on diagnostic and interventional cardiac catheterization, council on clinical cardiology, and the council on cardiovascular radiology and intervention: Endorsed by the American College of Cardiology Foundation, the North American Society for Cardiac Imaging, and the Society for Cardiovascular Magnetic Resonance. Circulation. 2007;116:2878-91.

17. Okumura W, Iwasaki T, Toyama $T$, et al. Usefulness of fasting 18F-FDG PET in identification of cardiac sarcoidosis. J Nucl Med. 2004;45:1989-98.

18. Youssef G, Leung E, Mylonas I, et al. The use of 18F-FDG PET in the diagnosis of cardiac sarcoidosis: A systematic review and metaanalysis including the Ontario experience. J Nucl Med. 2012;53:241-8.

19. Soejima K, Stevenson WG. Ventricular tachycardia associated with myocardial infarct scar: A spectrum of therapies for a single patient. Circulation. 2002;106:1769.

20. Soejima K, Stevenson WG, Sapp JL, et al. Endocardial and epicardial radiofrequency ablation of ventricular tachycardia associated with dilated cardiomyopathy: The importance of low-voltage scars. J Am Coll Cardiol. 2004;43:1834-42.

21. Bardy GH, Lee KL, Mark DB, et al. Amiodarone or an implantable cardioverter-defibrillator for congestive heart failure. N Engl J Med. 2005;352:225-37.

\section{PEER REVIEW}

Not commissioned. Externally peer reviewed.

\section{CONFLICTS OF INTEREST}

The authors declare that they have no competing interests.

\section{FUNDING}

None

\section{ETHICS COMMITTEE APPROVAL}

Cleveland Clinic institutional review board and ethics committee. 
Figure 1: An example of ${ }^{18}$ FDG PET imaging in a patient with VT storm. ${ }^{18}$ FDG PET imaging is shown demonstrating perfusion defect with increased FDG uptake (perfusion metabolism mismatch) in the mid and distal right ventricular septum extending to the inferoseptal segment of the left ventricle suggesting inflammation. The corresponding clinical manifestation shown on the electrocardiogram is sustained ventricular tachycardia originating from this region. Whole body FDG PET imaging shows FDG avid hilar and mediastinal adenopathy

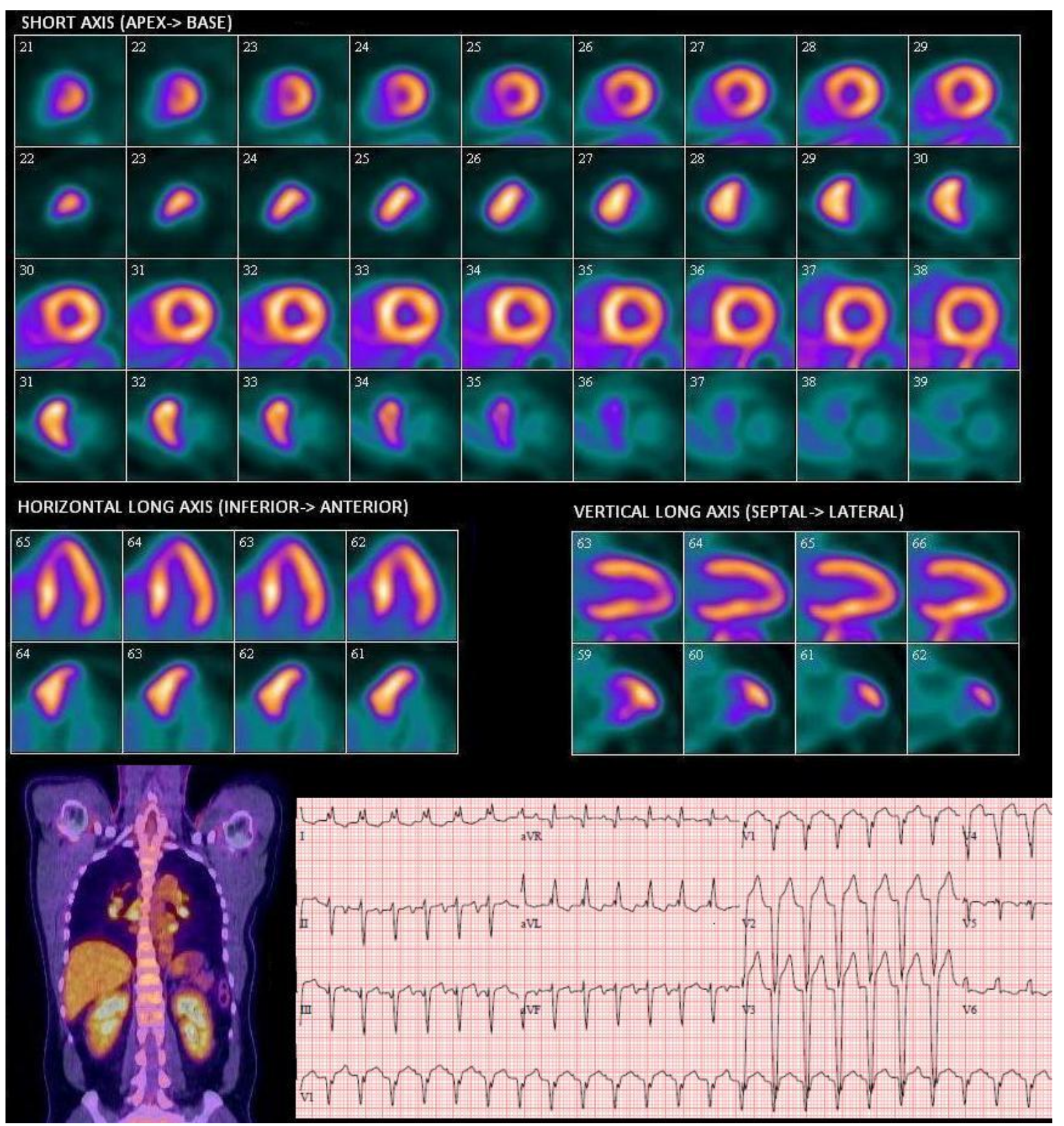


Figure 2: Prevalence of VT storm and conduction disease among patients with CS stratified by their regional ${ }^{18}$ FDG PET abnormalities. On the left, the bar graph shows the prevalence of VT storm in patients with and without regional inflammation in $\geq 2$ basal septal or mid septal segments. On the right, the bar graph shows the prevalence of advanced conduction disease in patients with and without inflammation or scar in the anteroseptal segment

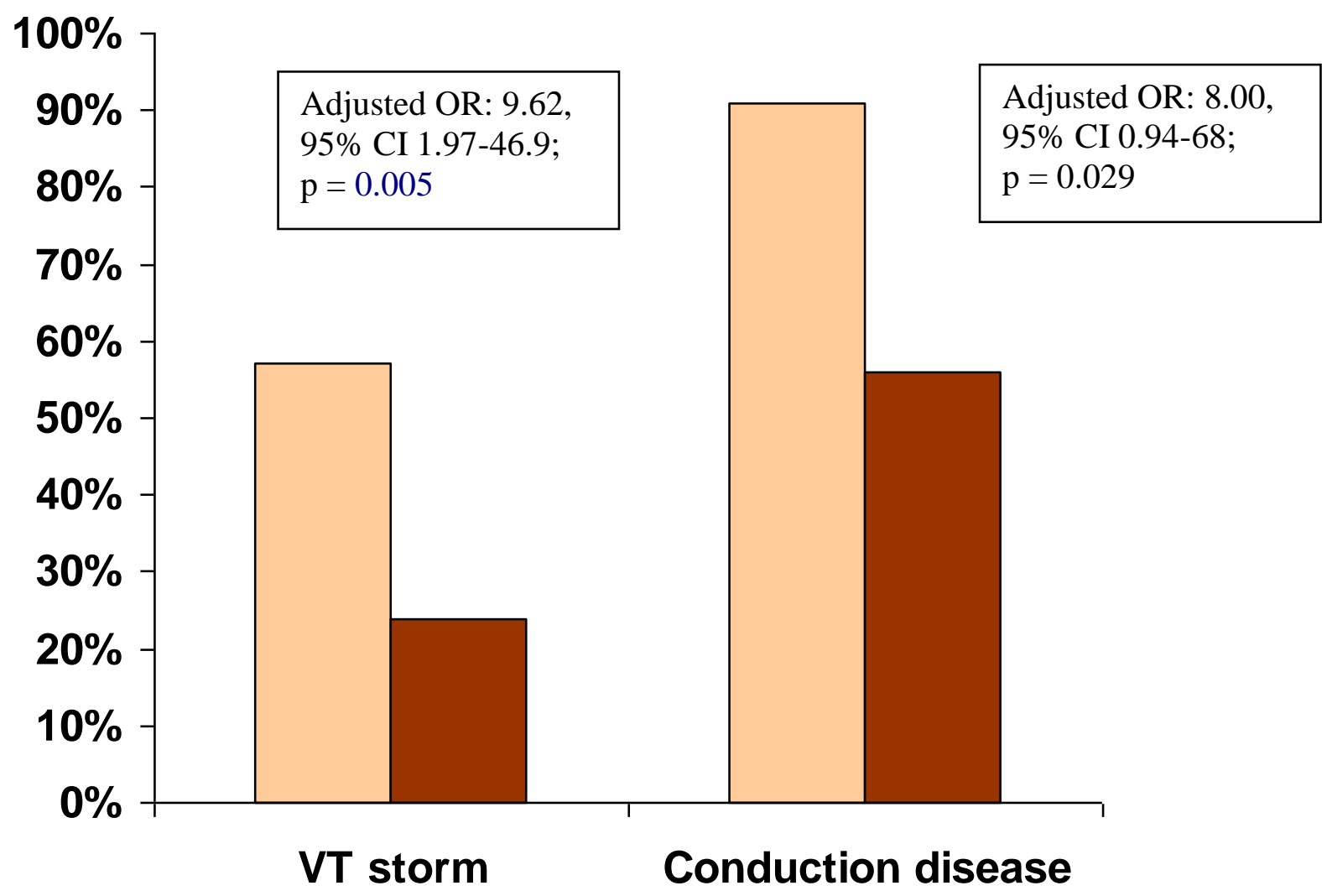

Figure 3: Receiver operating characteristic curve analysis of myocardial inflammatory burden on ${ }^{18}$ FDG PET for detecting VT storm. An area under the curve of 0.68 for $>15 \%$ inflammation burden on ${ }^{18}$ FDG PET imaging in the prediction of VT storm $(p<0.05)$ shown

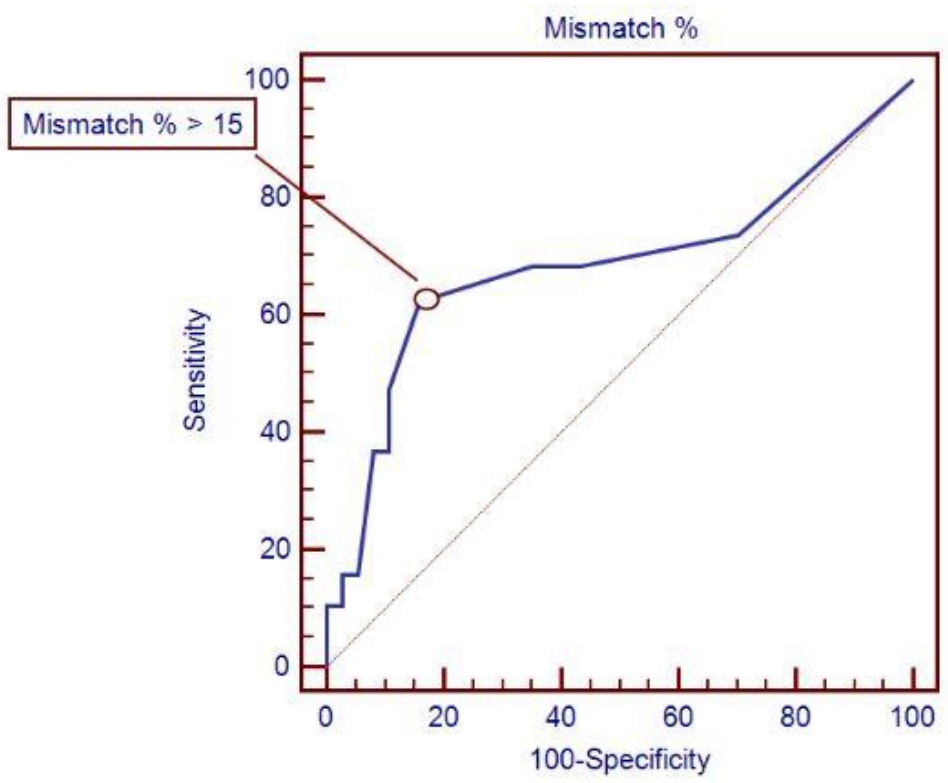


Figure 4: Probability of VT storm based on myocardial inflammatory burden on ${ }^{18}$ FDG PET by segments involved. Concordance between observed and predicted risk of VT storm. There is an incremental risk of VT storm with each subsequent inflammatory segment noted on ${ }^{18}$ FDG PET

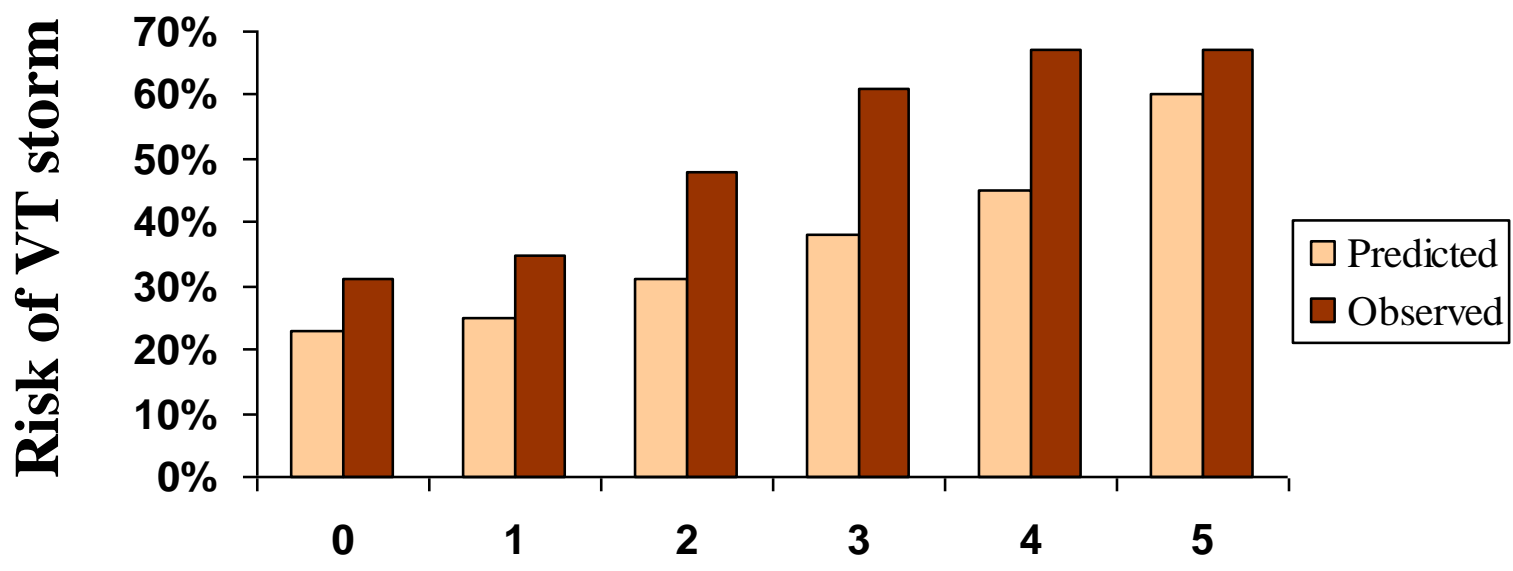

Number of mismatch segments

Table 1: Baseline characteristics and ${ }^{18}$ FDG PET findings of suspected CS patients

\begin{tabular}{|l|l|}
\hline Variable & (n=57) \\
\hline Age, years & $49 \pm 12$ \\
\hline Male gender & $29(51 \%)$ \\
\hline Caucasian race & $46(81 \%)$ \\
\hline Tobacco use & $17(30 \%)$ \\
\hline Diabetes mellitus & $6(11 \%)$ \\
\hline Left ventricular ejection fraction & $0.38 \pm 0.15$ \\
\hline Cardiac biopsy positive & $10(18 \%)$ \\
\hline Extra-cardiac biopsy positive & $37(65 \%)$ \\
\hline $\begin{array}{l}18 \text { FDG PET imaging } \\
\text { Normal }\end{array}$ & $1(2 \%)$ \\
\hline \multicolumn{1}{|l|}{ Scarlammation } & $17(30 \%)$ \\
\hline \multicolumn{1}{|c|}{ Combination } & $15(26 \%)$ \\
\hline Atrial fibrillation & $24(42 \%)$ \\
\hline Nonsustained VT & $18(32 \%)$ \\
\hline VT storm & $40(70 \%)$ \\
\hline Conduction disease & $19(33 \%)$ \\
\hline Complete heart block & $36(63 \%)$ \\
\hline
\end{tabular}

Data are expressed as mean value SD, or number of subjects (percentages) 
Table 2: Clinical characteristics of CS patients stratified by absence or presence of ventricular tachycardia storm

\begin{tabular}{|c|c|c|c|}
\hline Variable & $\begin{array}{l}\text { VT Storm } \\
(n=19)\end{array}$ & $\begin{array}{l}\text { No VT Storm } \\
(n=38)\end{array}$ & $P$ value \\
\hline Age, years & $49 \pm 10$ & $50 \pm 12$ & 0.788 \\
\hline Male gender & $9(47 \%)$ & $20(53 \%)$ & 0.708 \\
\hline Caucasian race & $16(84 \%)$ & 30 (79\%) & 0.635 \\
\hline Tobacco use & $6(32 \%)$ & $11(29 \%)$ & 0.838 \\
\hline Diabetes mellitus & $2(11 \%)$ & $6(11 \%)$ & 1 \\
\hline Left ventricular ejection fraction & $0.36 \pm 0.15$ & $0.40 \pm 0.15$ & 0.247 \\
\hline Cardiac biopsy positive & $7(37 \%)$ & $3(8 \%)$ & 0.031 \\
\hline Extra-cardiac biopsy positive & $7(37 \%)$ & $30(79 \%)$ & 0.024 \\
\hline \multicolumn{4}{|l|}{${ }^{18}$ FDG PET imaging } \\
\hline Normal & $0(0 \%)$ & $1(3 \%)$ & 0.476 \\
\hline Inflammation & $6(32 \%)$ & $11(29 \%)$ & 0.838 \\
\hline Scar & $5(26 \%)$ & $10(26 \%)$ & 1 \\
\hline Combination & $8(42 \%)$ & $16(42 \%)$ & 1 \\
\hline $\begin{array}{l}\text { Inflammation of } \geq 2 \text { basal or mid septal } \\
\text { segments }\end{array}$ & $9(47 \%)$ & $6(16 \%)$ & 0.011 \\
\hline Atrial fibrillation & $4(21 \%)$ & $14(37 \%)$ & 0.227 \\
\hline Conduction disease & $13(68 \%)$ & $23(61 \%)$ & 0.56 \\
\hline Complete heart block & $6(32 \%)$ & $8(21 \%)$ & 0.384 \\
\hline Left bundle branch block & $3(16 \%)$ & $8(21 \%)$ & 0.635 \\
\hline Right bundle branch block & $5(26 \%)$ & $12(32 \%)$ & 0.682 \\
\hline Class IB AAD (mexilitine) & $5(26 \%)$ & $1(3 \%)$ & 0.006 \\
\hline Class III AAD (sotalol, dofetilide) & $12(63 \%)$ & $8(21 \%)$ & 0.002 \\
\hline Amiodarone & $7(37 \%)$ & $2(5 \%)$ & 0.002 \\
\hline IV lidocaine or amiodarone & 19 (100\%) & $0(0 \%)$ & 0.003 \\
\hline \multicolumn{4}{|l|}{ Immunosuppression } \\
\hline Corticosteroids & $17(90 \%)$ & $32(84 \%)$ & 0.59 \\
\hline Methotrexate & $12(63 \%)$ & $20(53 \%)$ & 0.45 \\
\hline Azathioprine & $3(16 \%)$ & $7(18 \%)$ & 0.805 \\
\hline Leflunomide & $1(5 \%)$ & $5(13 \%)$ & 0.36 \\
\hline Mycophenolate & $2(11 \%)$ & $0(0 \%)$ & 0.042 \\
\hline Infliximab & $3(16 \%)$ & $8(22 \%)$ & 0.603 \\
\hline ACE inhibitor & $9(47 \%)$ & $14(37 \%)$ & 0.445 \\
\hline ARB & $4(21 \%)$ & $9(24 \%)$ & 0.823 \\
\hline Diuretics & $10(53 \%)$ & 20 (53\%) & 1 \\
\hline Beta blockers & 17 (90\%) & 30 (79\%) & 0.325 \\
\hline ICD & 19 (100\%) & 27 (71\%) & 0.009 \\
\hline
\end{tabular}

Data are expressed as mean value $\pm S D$, or number of subjects (percentages). ACE=angiotensin converting enzyme; $A R B=$ angiotensin receptor blocker; IV=intravenous 
Table 3: Clinical characteristics of CS patients stratified by absence or presence of conduction disease

\begin{tabular}{|c|c|c|c|}
\hline Variable & $\begin{array}{l}\text { Conduction } \\
\text { Disease }(n=36)\end{array}$ & $\begin{array}{l}\text { No Conduction } \\
\text { Disease }(n=21)\end{array}$ & $P$ value \\
\hline Age, years & $48 \pm 14$ & $47 \pm 15$ & 0.75 \\
\hline Male gender & $20(56 \%)$ & $9(43 \%)$ & 0.355 \\
\hline Caucasian race & $29(81 \%)$ & $17(81 \%)$ & 0.971 \\
\hline Tobacco use & $10(28 \%)$ & $7(33 \%)$ & 0.658 \\
\hline Diabetes mellitus & $3(8 \%)$ & $3(14 \%)$ & 0.48 \\
\hline Left ventricular ejection fraction & $0.37 \pm 0.16$ & $0.38 \pm 0.18$ & 0.549 \\
\hline Cardiac biopsy positive & $7(19 \%)$ & $3(14 \%)$ & 0.598 \\
\hline Extra-cardiac biopsy positive & $24(67 \%)$ & $13(62 \%)$ & 0.625 \\
\hline \multicolumn{4}{|l|}{${ }^{18}$ FDG PET imaging } \\
\hline Normal & $1(3 \%)$ & $0(0 \%)$ & 0.441 \\
\hline Inflammation & $8(22 \%)$ & $9(43 \%)$ & 0.1 \\
\hline Scar & $9(25 \%)$ & $6(29 \%)$ & 0.768 \\
\hline Combination & $18(50 \%)$ & $6(29 \%)$ & 0.114 \\
\hline $\begin{array}{l}\text { Inflammation of } \geq 2 \text { anteroseptal } \\
\text { segments }\end{array}$ & $10(28 \%)$ & $1(5 \%)$ & 0.034 \\
\hline Atrial fibrillation & $11(31 \%)$ & $7(33 \%)$ & 0.828 \\
\hline Nonsustained VT & $25(69 \%)$ & 15 (71\%) & 0.874 \\
\hline VT storm & $13(36 \%)$ & $6(29 \%)$ & 0.56 \\
\hline Class IB AAD (mexilitine) & $6(17 \%)$ & $0(0 \%)$ & 0.048 \\
\hline Class III AAD (sotalol, dofetilide) & $12(33 \%)$ & $8(38 \%)$ & 0.735 \\
\hline Amiodarone & $7(19 \%)$ & $2(10 \%)$ & 0.322 \\
\hline IV lidocaine or amiodarone & $13(36 \%)$ & $6(29 \%)$ & 0.56 \\
\hline \multicolumn{4}{|l|}{ Immunosuppression } \\
\hline Corticosteroids & $31(86 \%)$ & $18(86 \%)$ & 0.967 \\
\hline Methotrexate & $18(50 \%)$ & $14(67 \%)$ & 0.221 \\
\hline Azathioprine & $5(14 \%)$ & $5(24 \%)$ & 0.342 \\
\hline Leflunomide & $3(8 \%)$ & $3(14 \%)$ & 0.48 \\
\hline Infliximab & $8(22 \%)$ & $3(15 \%)$ & 0.515 \\
\hline ACE inhibitor & $15(42 \%)$ & $8(38 \%)$ & 0.791 \\
\hline ARB & $7(19 \%)$ & $6(29 \%)$ & 0.428 \\
\hline Diuretics & $17(47 \%)$ & $13(62 \%)$ & 0.284 \\
\hline Beta blockers & $30(83 \%)$ & 17 (81\%) & 0.82 \\
\hline Dual chamber pacemaker & $33(92 \%)$ & $16(76 \%)$ & 0.105 \\
\hline ICD & $31(86 \%)$ & $15(71 \%)$ & 0.175 \\
\hline
\end{tabular}

Data are expressed as mean value $\pm S D$, or number of subjects (percentages). ACE=angiotensin converting enzyme; $\mathrm{ARB}=$ angiotensin receptor blocker; IV=intravenous 